\title{
Improved antibody-guided surgery with a near-infrared dye on a PEGylated linker for CEA-positive tumors
}

Paul J. Yazaki

Thinzar M. Lwin

Megan Minnix

Lin $\mathrm{Li}$

Anakim Sherman

Justin Molnar

Aaron Miller

Paul Frankel

Junie Chea

Erasmus Poku

Nicole Bowles

Robert M. Hoffman

John E. Shively

Michael Bouvet

Paul J. Yazaki, Thinzar M. Lwin, Megan Minnix, Lin Li, Anakim Sherman, Justin Molnar, Aaron Miller, 


\title{
Improved antibody-guided surgery with a near-infrared dye on a PEGylated linker for CEA-positive tumors
}

\author{
Paul J. Yazaki, ${ }^{\mathrm{a}, \star}$ Thinzar M. Lwin, ${ }^{\mathrm{b}}$ Megan Minnix, ${ }^{\mathrm{a}}$ Lin Li, ${ }^{\mathrm{a}}$ Anakim Sherman, ${ }^{\mathrm{a}}$ Justin Molnar, ${ }^{\text {a }}$ Aaron Miller, ${ }^{\mathrm{a}}$ \\ Paul Frankel, ${ }^{c}$ Junie Chea, ${ }^{a}$ Erasmus Poku, ${ }^{a}$ Nicole Bowles, ${ }^{a}$ Robert M. Hoffman, ${ }^{b, d, e}$ John E. Shively, ${ }^{a}$ and \\ Michael Bouvet ${ }^{\text {b,e }}$ \\ aBeckman Research Institute, Diabetes Metabolism Research Institute, Department of Molecular Imaging and Therapy, City of Hope, \\ Duarte, California, United States \\ bUniversity of California San Diego, Department of Surgery, La Jolla, California, United States \\ 'Beckman Research Institute, Department of Computational and Quantitative Medicine, City of Hope, Duarte, California, United States \\ ${ }^{\mathrm{d} A n t i C a n c e r, ~ I n c ., ~ S a n ~ D i e g o, ~ C a l i f o r n i a, ~ U n i t e d ~ S t a t e s ~}$ \\ 'VA San Diego Healthcare System, San Diego, California, United States
}

\begin{abstract}
Real-time intraoperative image-guided cancer surgery promises to improve oncologic outcomes. Tumor-specific antibodies conjugated with near-infrared (NIR) fluorophores have demonstrated the potential to enhance visualization of solid tumor margins and metastatic disease; however, multiple challenges remain, including improvement in probe development for clinical utility. We have developed an NIR-IR800 dye on a PEGylated linker (sidewinder) conjugated to the humanized anti-carcinoembryonic antigen (CEA) antibody (M5A) with extended in vivo serum and tumor persistence. The anti-CEA M5A-sidewinder has a high dyeto-antibody ratio (average of 7 per antibody) that allows, in an orthotopic implanted human pancreatic cancer mouse model increased tumor fluorescence, higher tumor-to-background ratio and extends the surgical scheduling window compared to current antibody dye conjugates. These preclinical results demonstrate the potential of this probe for fluorescence-guided surgery of CEA-positive gastrointestinal cancers. $\odot$ The Authors. Published by SPIE under a Creative Commons Attribution 4.0 Unported License. Distribution or reproduction of this work in whole or in part requires full attribution of the original publication, including its DOI. [DOI: 10.1117/1.JBO.24.6.066012]
\end{abstract}

Keywords: fluorescence-guided-surgery; IR800 dye; anti-CEA antibody; polyethylene glycol; pancreatic cancer.

Paper 190027R received Jan. 30, 2019; accepted for publication Jun. 4, 2019; published online Jun. 28, 2019.

\section{Introduction}

Fluorescence-guided surgery (FGS) using tumor-specific antibodies conjugated with near-infrared fluorophores (NIR) have demonstrated the potential to visualize solid tumor lesions in preclinical and early stage clinical trials. ${ }^{1-3}$ Several commercial cancer therapeutic monoclonal antibodies (mAbs) have been repurposed as molecular optical imaging agents by their conjugation with NIR dyes and are in early-stage clinical trials. ${ }^{4-6}$ Compared to visible light fluorophores, NIR fluorophores have reduced light scattering, increased depth of penetration, and minimal autofluorescence. The use of NIR dyes in the 800$\mathrm{nm}$ range enables the use of instrumentation available in most operating suites using the FDA-approved indocyanine green dye to monitor cardiac output and liver blood flow function. Our theranostics group has focused on the development of anticarcinoembryonic antigen (CEA) mAbs for the radionuclide imaging and therapy of CEA-positive disease. ${ }^{7-12}$ CEA is surface GPI-linked glycoprotein highly expressed in gastrointestinal malignancies (GI), such as colorectal (95\%), gastric (80\%), pancreatic $(60 \%)$, and breast $(50 \%)$ cancers but with very limited expression in normal adults. ${ }^{13-17}$ We designed and produced a recombinant humanized anti-CEA hT84.66-M5A (M5A) mAb that is currently in early phase clinical trials. ${ }^{18}$ Radiolabeled with the positron emission tomography (PET) radionuclide $\mathrm{Cu}-64$, the ${ }^{64} \mathrm{Cu}$-DOTA-M5A $\mathrm{mAb}$ has provided promising PET

*Address all correspondence to Paul J. Yazaki, E-mail: pyazaki@coh.org images of CEA-positive disease in an ongoing pilot imaging trial (NCT02293954). Based on success with immunoPET imaging prior to surgery, a natural extension of the anti-CEA M5A $\mathrm{mAb}$ would be its development for fluorescent image-guided surgery. We have collaborated with the Bouvet group who have a long-standing expertise on the preclinical use of a chimeric anti-CEA antibody for FGS. ${ }^{19-23}$ Using the commercial NHSIRDye800CW currently being used in antibody-targeted FGS clinical trials, ${ }^{5,24}$ the M5A-IR800 exhibited strong tumor-specific labeling in a human colon cancer ${ }^{25}$ as well as pancreatic cancer $^{26}$ orthotopic xenograft mouse models. However, these studies identified room for improvement, in that conjugation of the IRDye800CW fluorophore to M5A resulted in accelerated pharmacokinetics over the unmodified M5A mAb, requiring low antibody-to-dye ratios, limiting sensitivity of tumor detection over a shortened window for surgical planning.

Addressing this issue, we developed an antibody-dye conjugate, anti-CEA "M5A-IR800 sidewinder," that incorporates a polyethylene glycol (PEG) linker with a sidearm for dye attachment to mask the dye's effect on clearance, thus extending its in vivo serum and tumor persistence. In proof of concept studies, the M5A-IR800 sidewinder exhibited exceptional optical imaging properties including a longer serum half-life compared to current antibody-dye conjugates, increased tumor fluorescence, with a higher tumor-to-background ratio, and importantly, an expanded window for surgical planning. The antiCEA M5A-IR800 sidewinder exemplifies the next-generation molecular-targeted optical agent for real-time image-guided GI cancer surgery. 


\section{Results}

Since many NIR dyes are supplied as active esters suitable for conjugation to protein surface amines, our previous studies began with conjugating the Licor NHS-IRDye $800 \mathrm{CW}$ dye to the humanized anti-CEA hT84.66-M5A (M5A) monoclonal antibody (M5A-IRDye800CW). Noninvasive and post-laparoscopic NIR optical imaging was performed in CEA-positive orthotopic implanted colorectal and pancreatic cancer models. When M5A-IRDye800CW was injected intravenously, and imaging performed over a 72-h time course, in both human colorectal HT29 and pancreatic Bx-PC3 cancer models the primary tumor and metastatic disease was well visualized over the normal tissue background. ${ }^{25,26}$ However, we noted that M5AIRDye800CW was cleared from the blood stream by $\sim 24 \mathrm{~h}$, compared to the parental antibody's clearance that exceeded 96 h. ${ }^{26}$ Based on other studies, ${ }^{27}$ it seemed likely that the altered biodistribution was due to the amount of dye conjugated to antibody. Since NHS conjugation chemistry is notoriously inefficient, the excess of NHS-dye over antibody must be strictly controlled.

To more fully explore this possibility, a number of M5AIR800 conjugates were synthesized using NHS-chemistry at increasing conjugation molar ratios of 10, 20, and 30:1 (dye to $\mathrm{mAb}$; Fig. 1). To determine the blood clearance rate, the antibody-dye conjugates were radiolabeled with Iodine-125, and intravenous injected into nontumor bearing mice. Figure 2 shows the M5A-IR800 at the lowest chemical conjugation ratio (10:1) exhibits blood clearance similar to the unconjugated antibody, whereas higher conjugation ratios resulted in rapid blood clearance. Mass spectrometry analysis determined the 10:1 conjugation ratio gave an M5A-IR800 with an average of three dyes per mAb, while the 20:1 had 4.6 dyes per mAb (Fig. S1 in the Supplementary Material). The accelerated plasma clearance observed with increasing number of dyes per $\mathrm{mAb}$ is similar to what has been observed for increased number of drugs per antibody in antibody drug conjugates (ADC) ascribed to the hydrophobic nature of the monomethyl auristatin $\mathrm{E}$ drug. ${ }^{27}$ This recent publication on ADC linkers have shown that PEGylation can extend the ADC pharmacokinetics of hydrophobic drug payloads and a lysine sidearm attachment site may be a preferred conformation. In addition, PEGylation has been a

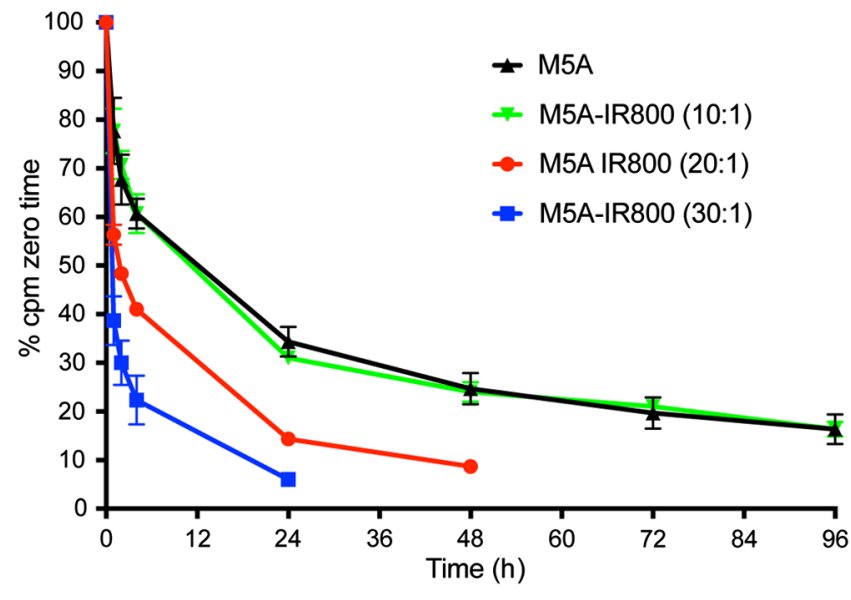

Fig. 2 Blood clearance of M5A-IR800 at varying conjugation ratios. The anti-CEA M5A mAb was conjugated with NHS-IR800 dye at conjugation ratios of 10,20, and 30:1 (dye NHS active ester to antibody). The antibody dye conjugates were radiolabeled with $\mathrm{I}-125$ and injected iv into athymic mice bearing human colorectal cancer LS174T xenografts. Blood was drawn at 0, 1, 2, 4, 24, and $48 \mathrm{~h}$, serum prepared, and counted for radioactivity. All data are reported as percent cpm zero time with standard errors.

tool to increase the in vivo plasma half-life extension of small molecular weight biopharmaceuticals. ${ }^{28}$

To investigate this approach, we utilized the commercially available reagent $\mathrm{NH}_{2}-\mathrm{dPEG}_{12}$-Lys (t-boc)-NH-dPEG (Quanta BioDesign, hereafter called "sidewinder"), consisting of monodispersed PEG units $\left(\mathrm{dPEG}^{\circledR}\right)$. Figure 3 shows the chemical steps used to generate a thiol site-specific PEGylateddye linker. The first step introduced a bromoacetamidol thiol reactive group to enable site-specific conjugation to reduced IgG1 hinge disulfides in order to sequester the linker-dye moiety in the hinge region of the antibody. The second step removed the tert-butyloxycarbonyl (t-boc) lysine-protecting group to enable conjugation of the dye NHS-active ester to the linker. The final product is designated as bromoacetamido-

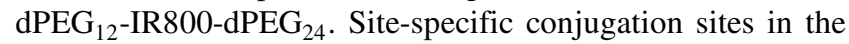
antibody were generated by mild reduction of the antibody with tris(2-carboxyethyl)phosphine (TCEP) as previously

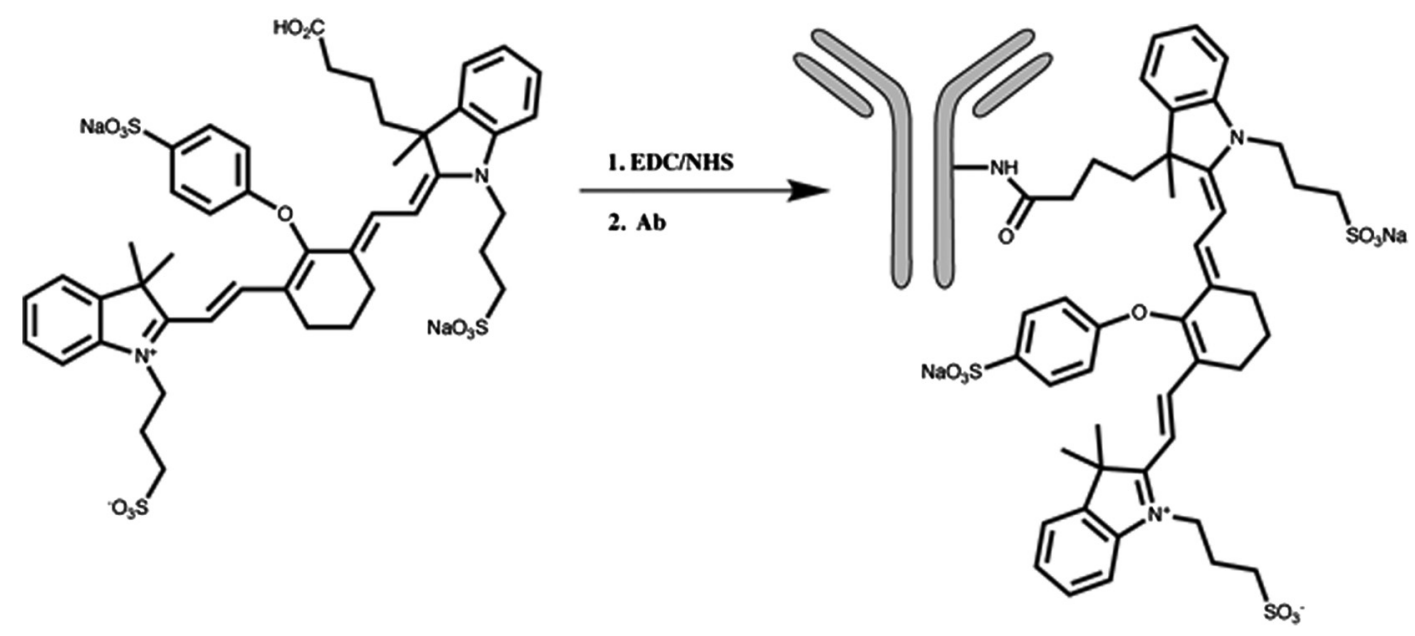

Fig. 1 Synthesis and conjugation of the active ester of IR800 dye to antibody. The IR800 active ester was synthesized by incubating the IR800 dye-free acid with NHS and EDC. The purified M5A antibody was then incubated with the NHS-IR800 dye at varying ratios. 
<smiles>CCCOCCOCCOCCNC(=O)C(CCCCCNC(=O)OC(C)(C)C)NC(=O)CCOCCC(C)(C)OCCOCC</smiles>

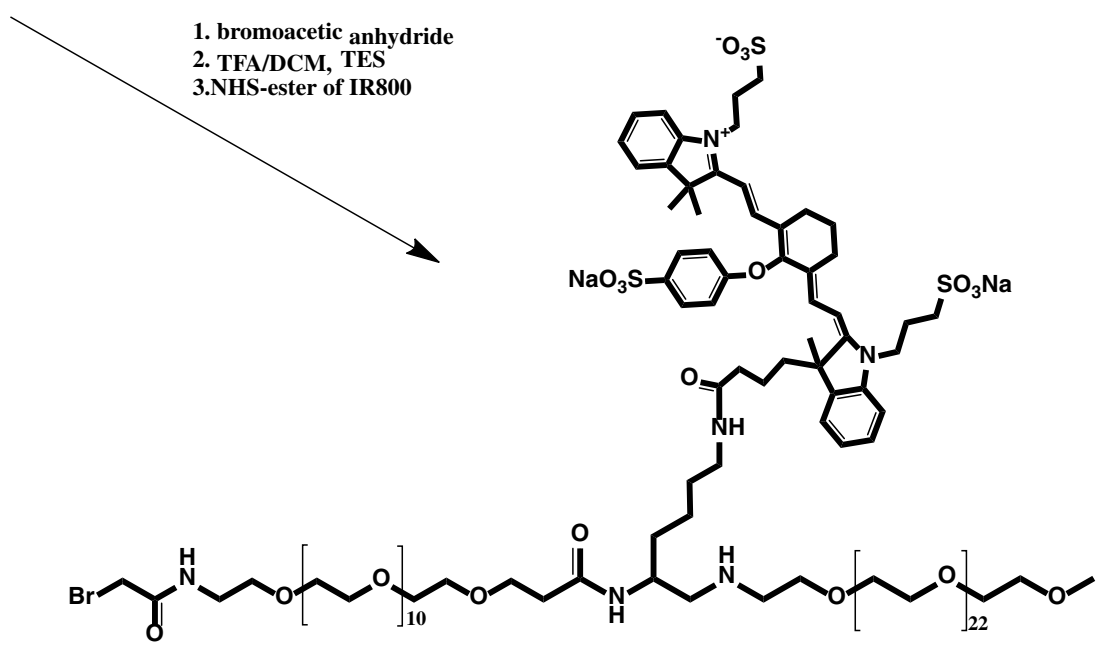

Fig. 3 Synthesis of bromoacetamido-PEG ${ }_{12}-I_{R} 800-P^{2} G_{24}$. The $\mathrm{NH}_{2}-\mathrm{dPEG}_{12}$-Lys(t-boc)-NH-m-dPEG ${ }_{24}$ was reacted with bromoacetic anhydride, the tert-butyloxycarbonyl (t-boc) protecting group removed and reacted with the NHS-ester of the IR800 dye.

described. ${ }^{29}$ The bromoacetamido-dPEG $12-\mathrm{IR}_{1200-\mathrm{dPEG}_{24} \text { was }}$ added to the reduced antibody at a molar ratio of $30: 1$. The M5A-IR800 sidewinder, as analyzed by mass spectroscopy indicated greater than six to seven dyes per antibody, close to complete modification of the eight available cysteines in the IgG1 hinge domain (Fig. S2 in the Supplementary Material). The M5A-sidewinder was analyzed by HPLC size exclusion chromatography (SEC) and exhibited a single peak that had increased molecular mass over the parental M5A antibody (Fig. S3 in the Supplementary Material).

To evaluate the tumor targeting and pharmacokinetic properties, the M5A-IR800 sidewinder and the parental anti-CEA M5A mAb were radiolabeled with Iodine-125 and injected intravenously into athymic mice bearing human colorectal cancer LS174T xenografts. Aliquots of blood were sampled over the 48-h time course. After the last sampling, the mice were euthanized, necropsy performed, tissues weighed, and counted for radioactivity enabling the data to be presented as the percent injected dose per gram (\% ID/g). The blood clearance curves show the M5A-IR800 sidewinder had slightly slower blood clearance than the antibody alone [Fig. 4(a)]. The biodistribution data at $48 \mathrm{~h}$ show the M5A-IR800 sidewinder had an average 4.3-fold increase in tumor accumulation over the M5A mAb with no substantial normal organ uptake [Fig. 4(b)].

In vivo NIR fluorescent imaging studies were performed with the M5A-IR800 and M5A-IR800 sidewinder in the human pancreatic cancer orthotopic mouse model previously described. ${ }^{26}$
The M5A-IR800 or M5A-IR800 sidewinder (75 $\mu \mathrm{g}$ dose) was injected intravenously via the tail vein. Individual mice were sacrificed and intravital imaging performed at 24, 48, 72, and $96 \mathrm{~h}$ on a Maestro CRI imaging system using the IR800 filter set. Figure 5 shows both anti-CEA antibody-dye conjugates were able to visualize tumors at the initial imaging timepoint at $24 \mathrm{~h}$ and that the fluorescence signal persisted at the tumor site throughout the 96-h experiment.

Quantification of the fluorescent signal intensity was recorded and tissue biodistribution plotted versus time (Fig. 6), and the M5A-IR800 control had a tumor peak fluorescent signal at $48 \mathrm{~h}$ that slowly washed out, but limited normal tissue uptake [Fig. 6(a)]. The M5A-IR800 sidewinder had higher fluorescent signal at the tumor site extending throughout the $96 \mathrm{~h}$ [Fig. 6(b)]. Due to the M5A-IR800 sidewinder's higher signal intensity, liver and bowel uptake was observed at $24 \mathrm{~h}$ but rapidly washed out. Calculating the area of the tumor fluorescence intensity versus time showed the area under the curve (AUC, between 24 and 96) of the M5A-IR800 sidewinder is $47 \%$ higher than the AUC of M5A-IR800 ( $p<0.001$, two-sided) [Fig. 6(c)]. This is an estimate since the M5A-IR800 sidewinder did not reach its tumor accumulation half-life by $96 \mathrm{~h}$. This is an important property that can be interpreted to allow a significant gain in the surgery scheduling window allowing increase clinical utility.

We performed a dose optimization study to determine an effective intravenous dose of the M5A-IR800 and M5AIR800 sidewinder for fluorescent imaging in an orthotopic 


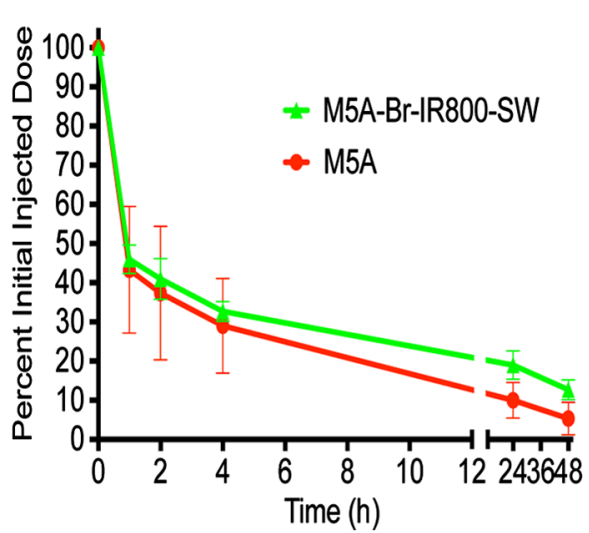

Blood clearance

(a)

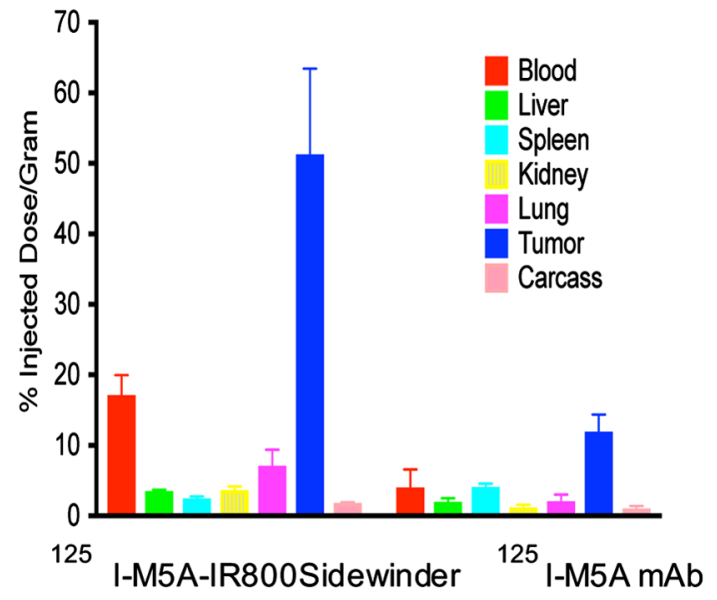

(b)

Fig. 4 Blood clearance and biodistribution of M5A-IR800 versus M5A-IR800-sidewinder. The parental anti-CEA M5A mAb and the M5A-IR800-sidewinder were radiolabeled with $\mathrm{I}-125$ and injected iv into athymic mice bearing human colorectal cancer LS174T xenografts. Blood was drawn at 0, 1, 2, 4, 24, and $48 \mathrm{~h}$. (a) The blood clearance was plotted versus time and (b) a tissue biodistribution performed at $48 \mathrm{~h}$. All data are mean values and have been corrected for radiodecay back to the time of injection, allowing uptake to be reported as percent injected dose per gram $(\% \mathrm{ID} / \mathrm{g})$ with standard error of mean.

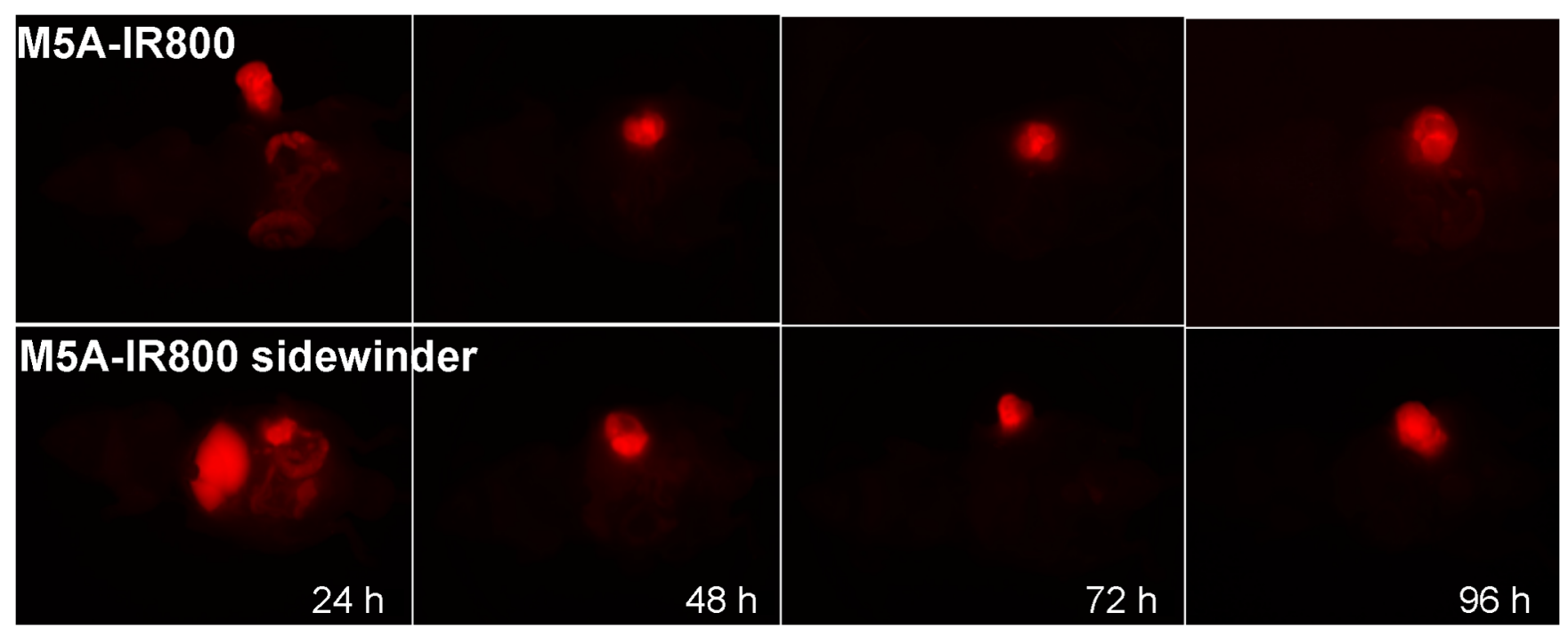

Fig. 5 M5A-IR800 and M5A-IR800 sidewinder in vivo NIR fluorescent imaging. The M5A-IR800 or M5AIR800 sidewinder ( $75 \mu \mathrm{g}$ dose) were injected into mice bearing orthotopic implanted human pancreatic cancer tumors. Individual mice were sacrificed and intravital NIR imaging performed at 24, 48, 72 , and $96 \mathrm{~h}$.

pancreatic cancer mouse model. The both conjugates were administered iv at 25,50 , and $75 \mu \mathrm{g}$ per mouse (three mice per group). Post-laparoscopic surgery, the mice were imaged at $48 \mathrm{~h}$. Both conjugates were able to visualize tumors at all dose levels with varying intensities (Fig. 7).

Plotting the tumor fluorescence intensity signal, both conjugates showed a dose response [Fig. 8(a)]. The M5A-IR800 sidewinder had a higher tumor signal appearing to approach a plateau while the M5A-IR800 continued to rise. For example, the M5A-IR800 sidewinder had 2.5- to 3-fold higher tumor signal at the 25 - and 50- $\mu$ g doses, which would allow a lower dose to be administered. Combined with tumor detection sensitivity, another key requirement for optical imaging is a high tumor:background ratio. Measuring normal tissue near the tumor site, the M5A-IR800 sidewinder exhibited higher tumor:background ratios than the M5A-IR800 at all dose levels [Fig. 8(b)]. The M5A-IR800 sidewinder 50- $\mu$ g dose had a tumor:background ratio of 24:1, a 1.75-fold improvement over the M5A-IR800 conjugate at $48 \mathrm{~h}$. Even the lowest M5AIR800 sidewinder dose tested at $25 \mu \mathrm{g}$ had a tumor:background ratio of $14: 1$.

\section{Summary and Discussion}

Antigen-specific fluorescent-guided surgery has demonstrated the potential to identify positive tumor margins and metastatic sites of disease in early stage clinical trials. ${ }^{1-3}$ Although there are 


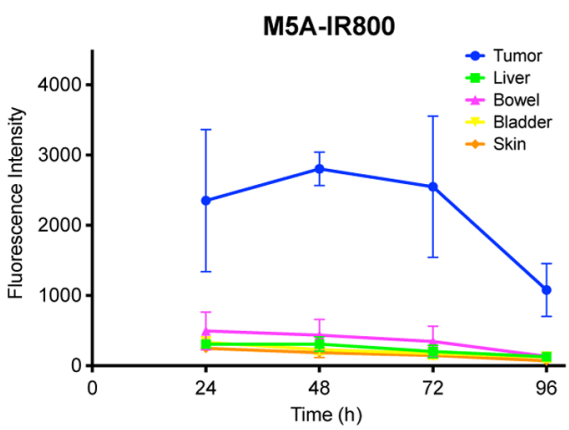

(a)

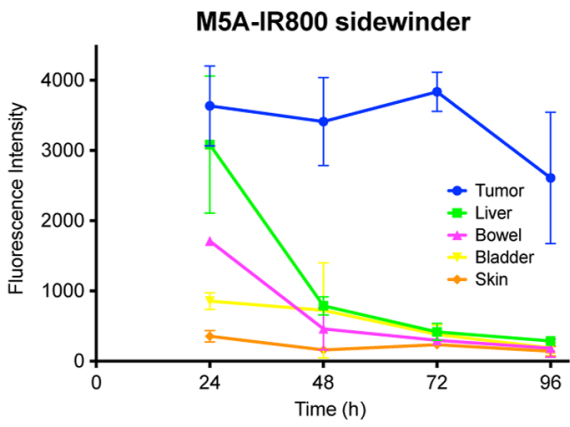

(b)

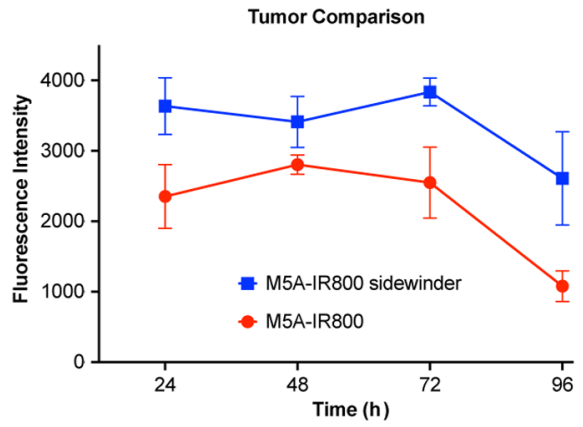

(c)

Fig. 6 M5A-IR800 and M5A-IR800 sidewinder in vivo fluorescent signal versus time. (a) The M5A-IR800 or (b) M5A-IR800 sidewinder fluorescent intensity signal from in vivo NIR fluorescent imaging (Fig. 3) was plotted for individual tissues versus time. (c) A comparison of the M5A-IR800 (red) or M5A-IR800 sidewinder (blue) AUC tumor accumulation was plotted.

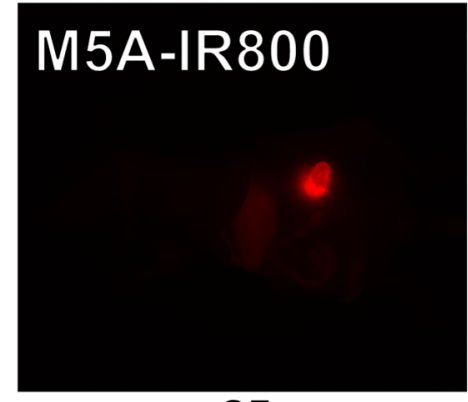

$25 \mu \mathrm{g}$

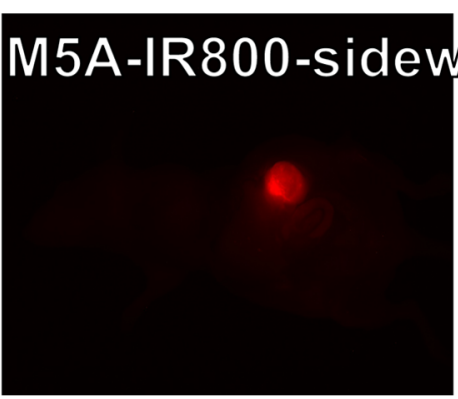

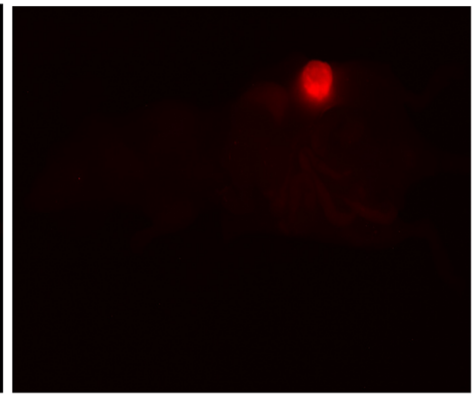

$50 \mu \mathrm{g}$

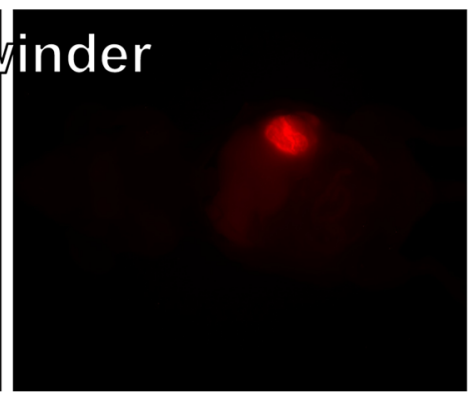

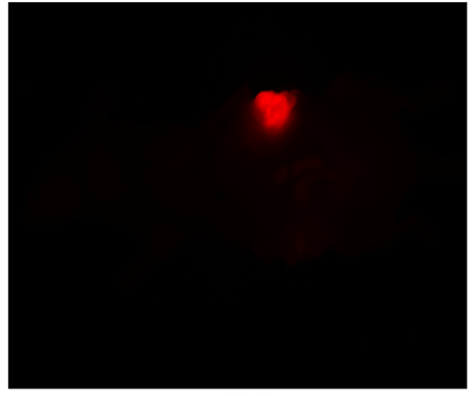

$75 \mu \mathrm{g}$

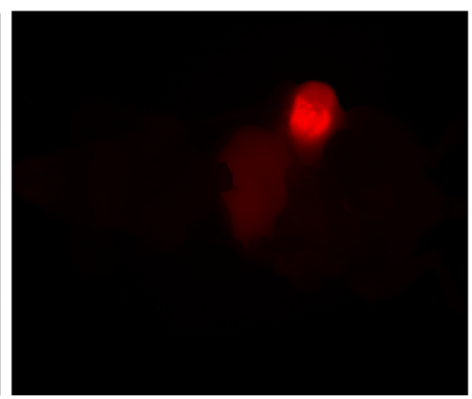

Fig. 7 M5A-IR800 and M5A-IR800 sidewinder dose optimization. The M5A-IR800 or M5A-IR800 sidewinder at doses of 25, 50, and $75 \mu \mathrm{g}$ were injected into mice bearing orthotopic implanted human pancreatic cancer tumors (three mice per dose per group). Individual mice were sacrificed and intravital imaging performed at $48 \mathrm{~h}$ on a Maestro CRI imaging system.

many challenges to advancing this promising field, we have focused on antitumor antibody-IR800 probe development to increase tumor accumulation and pharmacokinetic properties. Our previous work with M5A-IRDye800CW showed excellent tumor imaging but with high liver background and relatively fast blood clearance. ${ }^{25}$ In this report, we developed an anti-CEA tumor-targeted antibody-NIR IR800 fluorophore conjugate by synthesizing a unique PEGylated branched chain linker to potentially shield or mask the IR800 dye's hydrophobicity. An example of the M5A-IR800 sidewinder NIR imaging in an orthotopic pancreatic cancer model is shown in Fig. 9.

PEGylation is an established technology used to increase serum half-life extension for many small molecular-sized protein therapeutics by increasing their hydrodynamic size and radius, to increase resistance to proteases and potentially limit the generation of an immune response. ${ }^{30,31}$ In addition, PEG is a polyether, making it very hydrophilic. We postulate that these properties incorporated into a branched chain linker are important to overcome the fast serum clearance of an antibody highly conjugated with the highly hydrophobic IR800 dye. Future studies could determine the extent of mAb-sidewinder composition or conjugation required to tailor pharmacokinetics for specific applications as previously described for our anti-CEA diabody. ${ }^{32}$

Using site-specific thiol conjugation to the anti-CEA hT84.66-M5A IgG1 hinge domain, the M5A-IR800 sidewinder was able to achieve a high NIR signal (IR800 dye-to-antibody ratio $>6: 1$ ) without loss of antigen binding. This M5A-IR800 sidewinder exhibited enhanced in vivo serum half-life resulting in a dramatic increase in tumor accumulation compared to the 


\section{Dose Optimization}

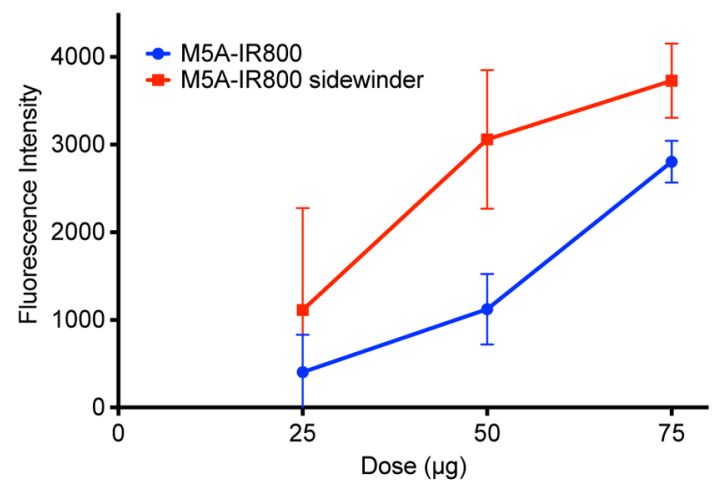

(a)

\section{Dose Optimization}

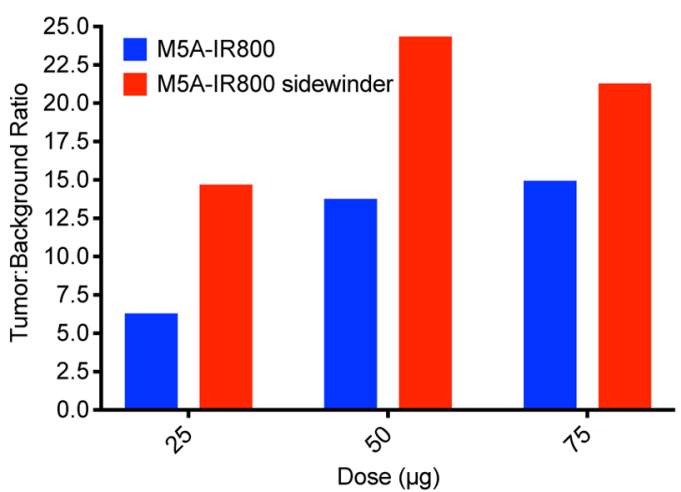

(b)

Fig. 8 M5A-IR800 and M5A-IR800 sidewinder dose optimization. (a) The M5A-IR800 (blue) and M5AIR800 sidewinder (red) fluorescent intensity versus dose was plotted from the in vivo NIR fluorescent imaging. (b) Tumor-to-background tissue fluorescent intensity was plotted versus dose.

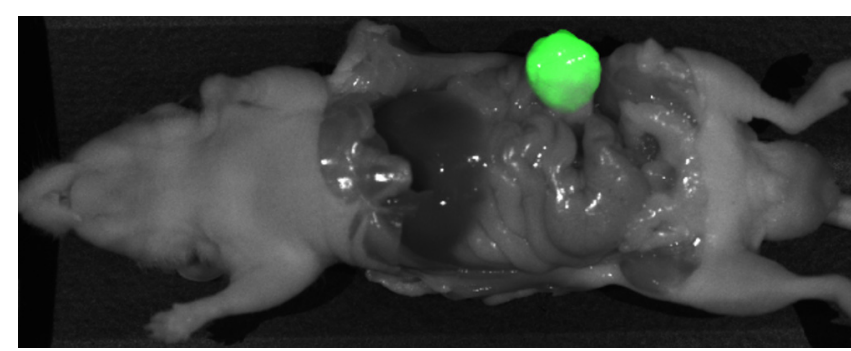

Fig. 9 NIR imaging of anti-CEA M5A-IR800 sidewinder in an orthotopic implanted human pancreatic cancer mouse model.

parental anti-CEA mAb as well as the NHS-IR800 mAb-conjugates in a mouse xenograft model. This led to tumor-tobackground ratios $(\mathrm{TBR})>14$ which is comparable to values obtained with our previous M5A-IRDye800CW construct. We were able to decrease the dose of the antibody-dye conjugate down to $25 \mu \mathrm{g}$ per animal while maintaining a bright fluorescence signal with high contrast (Figs. 7 and 8). A key issue for the clinical translation of antibody NIR dye conjugates has been the large amounts required to be administered to compensate for the fast blood clearance ${ }^{33}$

NIR-IR800 imaging studies in an orthotopic pancreatic cancer mouse model showed the M5A-IR800 sidewinder had higher fluorescent intensity signal at the tumor site that remained elevated throughout the 96-h study compared to a first-generation M5A-IR800 antibody-dye control. This increase in AUC has important ramifications to increase clinical utility. Our determination of the ideal surgical window is based on the operative time for most complex GI cancer operations that range from $2 \mathrm{~h}$ to over $5 \mathrm{~h}$. For clinical applicability and ease of use in practice, this imaging agent administered 1 to 2 days prior to surgery would give sufficient washout time to provide optimum TBR during the 2- to 5-h surgery. There are obvious disadvantages to prolonged circulation time, which include lower TBR at the time of surgery and the potential for false lymph node identification. Based on translation of our radiolabeled antibodies and antibody fragments, ${ }^{9,10,12}$ the mouse tumor targeting and PK studies are predictive to guide the optimized time of administration and dose for successful NIR imaging in first-in-human studies.
Alternative FGS approaches using anti-CEA mAbs are ongoing by several groups. ${ }^{34,35}$ SMG-101 an anti-CEA-IR700 conjugate in a phase 1 study shows feasibility to detect in CEA-expressing pancreatic cancer primary and metastases. ${ }^{35}$ However, this probe shows a TBR of 3.5 using a $30-\mu \mathrm{g}$ dose in animal models and TBR's between 1 and 1.5 in patients. ${ }^{35,36}$ Other antibody fluorophore conjugates for tumor-overexpressing antigens have been targeted such as EGFR and VEGF. ${ }^{6,24,33,37}$ As various FGS agents enter human surgical oncology studies, clinical utility will focus on best diseaserelated biomarkers, TBRs, depth of penetration, pharmacokinetics, biodistribution, instrumentation, and most importantly postsurgical overall survival.

We are currently performing IND enabling studies that include process development, cGMP manufacture, and toxicology studies to support an open-label, first-in-human phase I trial to evaluate the safety and biologic activity of the anti-CEA M5A-sidewinder in patients with CEA-positive colorectal cancer. The anti-CEA M5A-IR800 sidewinder holds promise to become the next-generation optical imaging agent, with high NIR fluorescence, extended PK, and widely applicable for intraoperative image-guided surgery in GI cancers as well as application for other CEA-positive adenocarcinomas.

\section{Methods}

\subsection{M5A-IR800 Antibody Conjugation}

The humanized M5A mAb was developed by grafting the CDR region of the murine anti-CEA antibody mT84.66 onto the humanized anti-p185HER2 antibody (Trastuzumab) framework as previously described. ${ }^{18}$ The IR800 active ester was synthesized by incubating the IR800 dye-free acid with sulfo- $N$ hydroxysuccinimide (NHS) and 1-ethyl-3-(3-dimethylaminopropyl)-carbodiimide (EDC) $\mathrm{pH} 7.4$ and stirred for $30 \mathrm{~min}$ at $4^{\circ} \mathrm{C}$ (Fig. 1). The purified M5A antibody in PBS was incubated with the NHS-IR800 dye at a 10-, 20-, and 30-fold molar excess of the esterified dye. The samples were rotated at room temperature under argon overnight, and then the conjugate was dialysis in PBS with five changes to remove excess dye. Concentrations of the M5A-IR800 conjugate were determined by absorbance ${ }_{280}$ 
and the antibody:dye ratio confirmed by Agilent 6520 QTOF mass spectrometry (Agilent Technologies, Santa Clara, California).

\subsection{Synthesis and Antibody Conjugation of $\mathrm{Br}^{-}-\mathrm{PEG}_{12}-\mathrm{IR} 800-\mathrm{PEG}_{24}$}

The reaction steps are shown in Fig. 3. $\mathrm{NH}_{2}-\mathrm{dPEG}_{12}-$ Lys(t-boc)-NH-m-dPEG 24 (305 mg, 0.16 mmol, MW 1916.32, Quanta BioDesign, Plain City OH, PN11810) was reacted with a twofold molar excess of bromoacetic anhydride (82.27 mg, $0.32 \mathrm{mmol}$ ) and 10-fold molar excess of solid sodium bicarbonate $\left(\mathrm{NaHCO}_{3}\right)(133.7 \mathrm{mg}, 1.59 \mathrm{mmol})$ in 6$\mathrm{ml}$ dimethylformamide (DMF) for $3 \mathrm{~h}$ to form $\mathrm{Br}-\mathrm{CH}_{2}-\mathrm{CO}$ $\mathrm{NH}_{2}-\mathrm{dPEG}_{12}$-Lys(t-boc)-NH-m-dPEG ${ }_{24}$. Compound 2 was purified using a CN prep HPLC column, buffer A $[0.1 \%$ trifluoroacetic acid (TFA) in $\mathrm{H}_{2} \mathrm{O}$ ] and buffer $\mathrm{B}$ (0.1\% TFA in $90 \%$ acetonitrile). The product was verified by ESI-MS (calculated 2037.27; observed 2037.36). The tert-butyloxycarbonyl (t-boc) protecting group was removed using 95\% TFA (6.65 ml), $2.5 \%$ dichloromethane (DCM) $(0.175 \mathrm{ml})$, and $2.5 \%$ triethylsilane (TES) $(0.175 \mathrm{ml})$ for $2 \mathrm{~h}$ at room temperature. The product was verified by ESI-MS (calculated 1937.15 and observed 1937.10). The NHS active ester of the IR800 dye (Fig. 3) (5.64 $\mathrm{mg}$ and $6.22 \mu \mathrm{mol}$ ) was reacted with the deprotected bromoacetamido product at a fourfold molar excess $(48.1 \mathrm{mg}$ and $0.025 \mathrm{mmol}$ ) over night at room temperature in $1-\mathrm{ml}$ reaction volume, the product purified by RP-HPLC using a Gemini RP column (46.9\% yield) and the mass verified by ESI-MS (calculated 2826.22 and observed 2829.54). The final product was conjugated to hinge region sulfhydryls on lightly reduced anti-CEA hT84.66-M5A antibody $(5.9 \mathrm{mg}, \quad 0.039 \mu \mathrm{mol}$; Lot\#11NOV16BS1). The antibody was reduced in a 1-ml reaction volume with a 30 -fold molar excess of TCEP $(0.338 \mathrm{mg}$ and $1.18 \mu \mathrm{mol})$ for $2 \mathrm{~h}$ at $37^{\circ} \mathrm{C}$, in $\mathrm{pH} 7.4 \mathrm{PBS}$ with $1-\mathrm{mM}$ ethylenediaminetetraacetic acid (EDTA) $(0.29 \mathrm{mg}$ and $1 \mu \mathrm{mol})$. The TCEP was removed with a spin column and immediately reacted with a 30-fold molar excess of the bromoacetamido-sidewinder dye (3.34 mg and $1.18 \mu \mathrm{mol})$ at room temperature overnight and extensively dialyzed to remove excess reagent. The mAbsidewinder dye product was analyzed by mass spectrometry using an Agilent 6520 QTOF to confirm conjugation and to calculate sidewinder dye antibody ratio (SDAR). The light chain was $75 \%$ conjugated with one dye sidewinder and the heavy chain was $100 \%$ conjugated with an average of three dye sidewinders. The calculated SDAR is 6.6. (Fig. S2 in the Supplementary Material). The product was further analyzed by SEC (Superdex 200 10/300 GL column, GE Healthcare). A complete shift in peak retention was observed, showing that $100 \%$ of the M5A antibody was conjugated with sidewinder dye (Fig. S3 in the Supplementary Material).

\subsection{Radiolabeling}

The M5A-IR800 and M5A-IR800-sidewinder were radioiodinated by the Iodogen method at a specific activity of $10 \mathrm{MCi}$ per mg and purified by SEC Superdex 200 10/30 GL column (GE Biosciences) as previously described. ${ }^{38}$ The purity of the radiolabeled $\mathrm{mAb}$ was determined by monitoring the radioactivity output by SEC.

\subsection{Tissue Culture}

The human pancreatic cancer cell lines BxPC-3 (ATCC ${ }^{\circledR}$ CRL$1687^{\mathrm{TM}}$ ), stably expressing green fluorescent protein (GFP) were maintained in Roswell Park Memorial Institute 1640 (RPMI-1640) medium (Gibco-BRL, Grand Island, New York). The medium was supplemented with $10 \%$ fetal calf serum (Hyclone, Logan, Utah), 1\% L-glutamine, and 1\% penicillin/ streptomycin (Gibco-BRL). The cells were incubated at $37^{\circ} \mathrm{C}$ in a $5 \% \mathrm{CO}_{2}$ incubator.

\subsection{Animal Studies}

\subsubsection{Radioactive animal studies}

Groups of 7- to 8-week-old female athymic mice (Charles River Laboratories, Wilmington, Massachusetts) were injected subcutaneously in the flank region with $10^{6}$ LS174T human colon carcinoma cells obtained from American Tissue Culture Center (ATCC, Manassas, Virginia). After 10 days, when tumor masses were in the range of 100 to $200 \mathrm{mg}$, two to three microcuries $(\mu \mathrm{Ci})$ of ${ }^{125} \mathrm{I}-\mathrm{M} 5 \mathrm{~A}-\mathrm{IR} 800,{ }^{125} \mathrm{I}$-M5A-IR800-sidewinder, or parental M5A antibody (three per group) were injected into the tail vein. At time points $0,1,2,4,24$, and $48 \mathrm{~h}$, blood was drawn, serum prepared, and an aliquot counted for radioactivity. For biodistribution, after the final blood draw, the mice were euthanized, necropsy performed, organs weighed, and counted for radioactivity. The radioactivity has been corrected for background and radioactive decay from the time of injection, allowing organ uptake to be reported as percent counts per minute $(\mathrm{cpm})$ zero time or percent injected dose per gram $(\%$ ID/g) with standard error of mean. All animal studies were conducted under City of Hope Institutional Animal Care and Use Committee (IACUC) approved protocols (IACUC \#15003 \& \#91037).

\subsubsection{NIR fluorescent animal studies}

Immunocompromised nude nu/fox mice were maintained in a barrier facility on high-efficiency particulate ai-filtered racks at AntiCancer Inc. Mice were maintained ad lib on an autoclaved laboratory rodent diet (Teckland LM-485; Western Research Products, Orange, California) and kept on a 12-h light/12-h dark cycle. All surgical procedures and intravital imaging were performed with the animals anesthetized by intramuscular injection of an anesthetic cocktail composed of ketamine $100 \mathrm{mg} / \mathrm{kg}$ (MWI Animal Health, Boise, Idaho), xylazine $10 \mathrm{mg} / \mathrm{kg}$ (VWR, Brisbane, California), and acepromazine $3 \mathrm{mg} / \mathrm{kg}$ (Sigma, Saint Louis, Missouri). All animal studies were conducted in accordance with the principles and procedures outlined in the NIH Guide for the Care and Use of Animals under PHS Assurance Number A3873-1.

\subsubsection{Surgical orthotopic implantation and in-vivo NIR fluorescent studies}

BxPC-3-GFP pancreatic cancer cells $\left(1 \times 10^{6}\right.$ cells per animal $)$ were injected subcutaneously into the flank of nude mice. The tumors were allowed to grow for 4 weeks or until 7 to $10 \mathrm{~mm}$ in size. The tumors were harvested and $2 \mathrm{~mm}^{3}$ fragments were implanted into the pancreatic tail of recipient nude mice to create orthotopic models of pancreatic cancer. ${ }^{26,39}$ After the tumors developed for 2 weeks, mice were separated into the M5AIR800 and M5A-IR800 sidewinder groups and injected 
intravenous via tail-vein for the various studies. ${ }^{17-19}$ For the imaging time curve, two to five mice per conjugate per time point were sacrificed and imaged at 24, 48, 72, and $96 \mathrm{~h}$ after injection. For the dose optimization and tissue:background ratio study, three mice per conjugate were administered doses of 25,50 , and $75 \mu \mathrm{g}$, sacrificed after $48 \mathrm{~h}$ and imaged. The images were acquired at the IRDye 800 wavelength (excitation $778 \mathrm{~nm}$ and emission $800 \mathrm{~nm}$ ) using the Maestro CRI imaging system (Perkin Elmer, Waltham, Massachusetts). Fluorescence intensity was quantified at the skin and tumor after spectral unmixing using the Maestro CRI software. The average fluorescence signal at the region-of-interest was obtained at the tumor and viscera. Fluorescence intensity was adjusted for background signal at the skin by subtracting the intensity value at an area of adjacent skin to the intensity value at the tumor. TBR was calculated by dividing the intensity value at an area of adjacent skin to the intensity value at the tumor.

\subsubsection{Statistical analysis}

The statistical test for the increase in AUC associated with the sidewinder was based on resampling (null hypothesis based on random resampling of intensities within each time point without regard to the construct) and using a histogram method to evaluate the frequency that the resampled relative difference exceeds, in magnitude, the observed $47 \%$ difference.

\section{Disclosures}

$\mathrm{RMH}$ is an unsalaried associate of Anticancer Inc., which offers PDOX models for contract research. There are no other competing financial interests.

\section{Acknowledgments}

We wish to thank Paul Davis, Quanta Biodesign, Plain City IL for the PEGylated reagent $\mathrm{NH}_{2}-\mathrm{dPEG}_{12}$-Lys(t-boc)-NH-dPEG $\mathrm{d}_{24}$. Research reported in this publication included work performed in the Small Animal Imaging Core supported by the U.S. National Cancer Institute of the National Institutes of Health under award number P30CA33572. The content is solely the responsibility of the authors and does not necessarily represent the official views of the National Institutes of Health. Additional support by U.S. National Cancer Institute, Grant Nos: CA126023, CA142669 (MB); VA Merit Review, Grant No: 1 IO1 BX003856-01A1 (MB); NIH/NCI T32CA121938 (TL). An earlier version of the manuscript was submitted as SPIE proceedings.

\section{References}

1. M. T. Olson, Q. P. Ly, and A. M. Mohs, "Fluorescence guidance in surgical oncology: challenges, opportunities, and translation," Mol. Imaging Biol. 21(2), 200-218 (2018).

2. P. S. Low, S. Singhal, and M. Srinivasarao, "Fluorescence-guided surgery of cancer: applications, tools and perspectives," Curr. Opin. Chem. Biol. 45, 64-72 (2018).

3. B. W. Pogue et al., "Perspective review of what is needed for molecularspecific fluorescence-guided surgery," J. Biomed. Opt. 23(10), 100601 (2018).

4. E. L. Rosenthal et al., "In vivo detection of head and neck cancer orthotopic xenografts by immunofluorescence," Laryngoscope 116(9), 1636-1641 (2006).

5. W. S. Tummers et al., "Intraoperative pancreatic cancer detection using tumor-specific multimodality molecular imaging," Ann. Surg. Oncol. 25(7), 1880-1888 (2018).
6. E. L. Rosenthal et al., "The status of contemporary image-guided modalities in oncologic surgery," Ann. Surg. 261(1), 46-55 (2015).

7. J. Y. Wong et al., "Dose escalation trial of indium-111-labeled anticarcinoembryonic antigen chimeric monoclonal antibody (chimeric T84.66) in presurgical colorectal cancer patients," J. Nucl. Med. 12, 2097-2104 (1998).

8. J. Y. Wong et al., "A Phase I trial of 90 Y-anti-carcinoembryonic antigen chimeric T84.66 radioimmunotherapy with 5-fluorouracil in patients with metastatic colorectal cancer," Clin. Cancer Res. 16, 5842-5852 (2003).

9. J. Y. Wong et al., "Pilot trial evaluating an 123I-labeled 80-kilodalton engineered anticarcinoembryonic antigen antibody fragment (cT84.66 minibody) in patients with colorectal cancer," Clin. Cancer Res. 10, 5014-5021 (2004).

10. J. Y. C. Wong et al., "Initial experience evaluating ${ }^{90}$ yttrium-radiolabeled anti-carcinoembryonic antigen chimeric T84.66 in a phase I radioimmunotherapy trial," Cancer Res. 55, 5929s-5934s (1995).

11. J. Y. C. Wong et al., "Clinical evaluation of indium-111-labeled chimeric anti-CEA monoclonal antibody," J. Nucl. Med. 38, 19511959 (1997).

12. J. Y. C. Wong et al., "Initial clinical experience evaluating yttrium-90chimeric T84.66 anticarcinoembryonic antigen antibody and autologous hematopoietic stem cell in patients with carcinoembryonic antigenproducing metastatic breast cancer," Clin. Cancer Res. 5, 3224-3231 (1999).

13. J. Kim et al., "Expression of carcinoembryonic antigen and related genes in lung and gastrointestinal cancers," Int. J. Cancer 52(5), 718-725 (1992).

14. J. Bhatnagar et al., "Immunohistochemical detection of carcinoembryonic antigen in esophageal carcinomas: a comparison with other gastrointestinal neoplasms," Anticancer Res. 22(3), 1849-1857 (2002).

15. J. W. Park et al., "Carcinoembryonic antigen as a predictor of pathologic response and a prognostic factor in locally advanced rectal cancer patients treated with preoperative chemoradiotherapy and surgery," Int. J. Radiat. Oncol. Biol. Phys. 74(3), 810-817 (2009).

16. F. Gebauer et al., "Carcinoembryonic antigen-related cell adhesion molecules (CEACAM) 1, 5 and 6 as biomarkers in pancreatic cancer," PLoS One 9(11), e113023 (2014).

17. S. W. de Geus et al., "Selecting tumor-specific molecular targets in pancreatic adenocarcinoma: paving the way for image-guided pancreatic surgery," Mol. Imaging Biol. 18(6), 807-819 (2016).

18. P. J. Yazaki et al., "Humanization of the anti-CEA T84.66 antibody based on crystal structure data," Protein Eng. Des. Sel. 17(5), 481489 (2004).

19. C. A. Metildi et al., "Fluorescently labeled chimeric anti-CEA antibody improves detection and resection of human colon cancer in a patientderived orthotopic xenograft (PDOX) nude mouse model," J. Surg. Oncol. 109(5), 451-458 (2014).

20. C. A. Metildi et al., "Fluorescence-guided surgery with a fluorophoreconjugated antibody to carcinoembryonic antigen (CEA), that highlights the tumor, improves surgical resection and increases survival in orthotopic mouse models of human pancreatic cancer," Ann. Surg. Oncol. 21(4), 1405-1411 (2014).

21. E. L. Rosenthal et al., "Successful translation of fluorescence navigation during oncologic surgery: a consensus report," J. Nucl. Med. 57(1), 144-150 (2016).

22. A. A. Maawy et al., "Polyethylene glycol (PEG) linked to near infrared (NIR) dyes conjugated to chimeric anti-carcinoembryonic antigen (CEA) antibody enhances imaging of liver metastases in a nude-mouse model of human colon cancer," PLoS One 9(5), e97965 (2014).

23. Y. Hiroshima et al., "Successful fluorescence-guided surgery on human colon cancer patient-derived orthotopic xenograft mouse models using a fluorophore-conjugated anti-CEA antibody and a portable imaging system," J. Laparoendosc. Adv. Surg. Tech. A 24(4), 241-247 (2014).

24. E. L. Rosenthal et al., "Safety and tumor specificity of cetuximabIRDye800 for surgical navigation in head and neck cancer," Clin. Cancer Res. 21(16), 3658-3666 (2015).

25. J. C. DeLong et al., "Near-infrared-conjugated humanized anti-carcinoembryonic antigen antibody targets colon cancer in an orthotopic nude-mouse model," J. Surg. Res. 218, 139-143 (2017). 
26. T. M. Lwin et al., "Tumor-specific labeling of pancreatic cancer using a humanized anti-CEA antibody conjugated to a near-infrared fluorophore," Ann. Surg. Oncol. 25(4), 1079-1085 (2018).

27. R. P. Lyon et al., "Reducing hydrophobicity of homogeneous antibodydrug conjugates improves pharmacokinetics and therapeutic index," Nat. Biotechnol. 33(7), 733-735 (2015).

28. S. B. van Witteloostuijn, S. L. Pedersen, and K. J. Jensen, "Half-life extension of biopharmaceuticals using chemical methods: alternatives to PEGylation," ChemMedChem 11(22), 2474-2495 (2016).

29. Z. Zhou et al., "Specific conjugation of the hinge region for homogeneous preparation of antibody fragment-drug conjugate: a case study for doxorubicin-PEG-anti-CD20 Fab' synthesis," Bioconjug. Chem. 27(1), 238-246 (2016).

30. A. D. AlQahtani et al., "Strategies for the production of long-acting therapeutics and efficient drug delivery for cancer treatment," Biomed. Pharmacother. 113, 108750 (2019).

31. Z. Hussain et al., "PEGylation: a promising strategy to overcome challenges to cancer-targeted nanomedicines: a review of challenges to clinical transition and promising resolution," Drug Deliv. Transl. Res. 9(3), 721-734 (2019).

32. $\mathrm{L}$. Li et al., "Improved biodistribution and radioimmunoimaging with poly(ethylene glycol)-DOTA-conjugated anti-CEA diabody," Bioconjug. Chem. 17(1), 68-76 (2006).

33. E. L. Rosenthal et al., "Sensitivity and specificity of cetuximabIRDye $800 \mathrm{CW}$ to identify regional metastatic disease in head and neck cancer," Clin. Cancer Res. 23(16), 4744-4752 (2017).
34. M. C. H. Hekman et al., "Detection of micrometastases using SPECT/ fluorescence dual-modality imaging in a CEA-expressing tumor model," J. Nucl. Med. 58(5), 706-710 (2017).

35. C. E. S. Hoogstins et al., "Image-guided surgery in patients with pancreatic cancer: first results of a clinical trial using SGM-101, a novel carcinoembryonic antigen-targeting, near-infrared fluorescent agent," Ann. Surg. Oncol. 25(11), 3350-3357 (2018).

36. M. Gutowski et al., "SGM-101: an innovative near-infrared dyeantibody conjugate that targets CEA for fluorescence-guided surgery," Surg. Oncol. 26(2), 153-162 (2017).

37. A. G. Terwisscha van Scheltinga et al., "Intraoperative near-infrared fluorescence tumor imaging with vascular endothelial growth factor and human epidermal growth factor receptor 2 targeting antibodies," J. Nucl. Med. 52(11), 1778-1785 (2011).

38. A. M. Wu et al., "Tumor localization of anti-CEA single-chain FVS: improved targeting by noncovalent dimmers," Immunotechnology $\mathbf{2}$, 21-36 (1996).

39. M. Bouvet et al., "Chronologically-specific metastatic targeting of human pancreatic tumors in orthotopic models," Clin. Exp. Metastasis 18(3), 213-218 (2000).

Biographies of the authors are not available. 